\title{
Professor Gerhart Hitzenberger zum 85. Geburtstag
}

\author{
Herbert Kurz · Johannes G. Meran · Peter Pietschmann
}

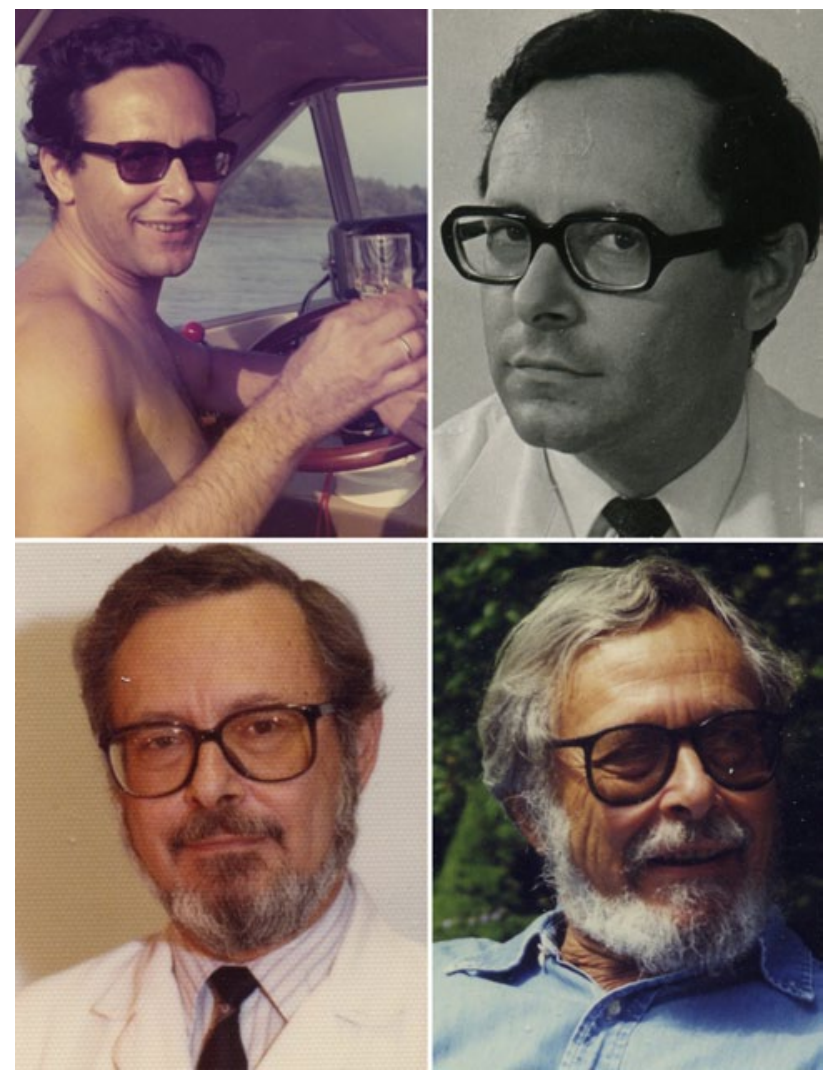

Prim. Dr. H. Kurz ( $\square)$

Abteilung für Kinder- und Jugendheilkunde,

Sozialmedizinisches Zentrum Ost - Donauspital,

Langobardenstraße 122, 1220 Wien, Österreich

E-Mail: herbert.kurz@wienkav.at

Prim. Univ.-Prof. Dr. J. G. Meran

Innere Medizin, Krankenhaus der Barmherzigen Brüder Wien, Wien, Österreich

Univ.-Prof. Dr. P. Pietschmann

Institut für Pathophysiologie und Allergieforschung,

Med. Universität Wien, Wien, Österreich
1978 übernahm Professor Gerhart Hitzenberger gemeinsam mit Professor Gerhard S. Barolin und Professor Heinz Sterz die Chefredaktion der Wiener Medizinischen Wochenschrift (WMW) und hat über viele Jahre die Geschicke der WMW gelenkt. Seit dem Jahr 2000 ist er ein aktiver Ehren-Herausgeber, der nach wie vor regen Anteil an den Aktivitäten der Herausgeber nimmt.

Seine großen Verdienste um die klinische Pharmakologie, seine wissenschaftlichen Leistungen und seine hohe Reputation im In- und Ausland sind Gegenstand von Ehrungen und Auszeichnungen.

Zur traditionsreichen Wiener Medizinische Wochenschrift kam Professor Hitzenberger zunächst 1977 als Redakteur der „Therapeutischen Erfahrungsberichte“, in denen neue Pharmaka und Therapieoptionen beleuchtet und den anwendenden Ärzten nähergebracht wurden. 1978 übernahm Professor Hitzenberger gemeinsam mit Professor Barolin und Professor Sterz die Chefredaktion der $W M W$. Der Erfolg dieses Triumvirats für die Zeitschrift war begründet, einerseits in enger Zusammenarbeit, andererseits in der regionalen Aufteilung (Prof. Hitzenberger in Wien, Prof. Sterz in Graz und Prof. Barolin in Vorarlberg), welche die Wiener Medizinische Wochenschrift $\mathrm{zu}$ einem gesamtösterreichischen Fortbildungsmedium machte.

Professor Hitzenberger baute das Begutachtungssystem aus und koordinierte die eintreffenden Arbeiten. Als Reviewer war und ist er von unbestechlicher Klarheit, was Qualität und Form der Manuskripte anlangt, und alle, die in der $W M W$ publiziert haben, schätzen seine sachliche, immer höfliche und nie verletzende Kritik.

Gemeinsam mit seinen Kollegen verfolgte Professor Hitzenberger das Konzept der gehobenen Fortbildung. In einer immer spezialisierteren Medizin bedarf es der verdichteten und übersichtlichen Zusammenfassungen, der Vermittlung von wissenschaftlichen Neuigkeiten an den praktizierenden Arzt. Themenhefte, die den neuesten Stand in einem Spezialgebiet zusammenfassen, 
waren und sind erfolgreiche und gefragte Publikationen der $W M W$, von denen Professor Hitzenberger sehr viele selbst gestaltete, akquirierte und anregte.

Das aktuelle Herausgeberteam hat dieses Konzept fortgesetzt und damit viele erfolgreiche Hefte gestaltet.

Entscheidend ist der wissenschaftliche Anspruch von Studien, aber auch von Übersichten und Fallberichten. Diesen wissenschaftlichen Anspruch hat Professor Hitzenberger über Jahrzehnte vorgemacht, gelehrt und als Reviewer und Herausgeber überwacht. Besonders hinzuweisen ist in diesem Zusammenhang auf seinen großen Verdienst um die Etablierung von Ethikkommissionen, ohne deren Votum heute keine Studie begonnen wird und auch keine publiziert werden kann.

Das Hauptmotiv seiner ärztlichen Tätigkeit war immer der individuelle Mensch in seinem Leiden. Dies geht zusammen mit einer Haltung von Demut (vor vielem Leid, das wir nach wie vor nicht lindern können), Bescheidenheit und großer Offenheit. Dies hat auch uns jüngere Herausgeber der $W M W$ beeinflusst und geprägt.

Beeindruckt hat uns neben seiner von Humanität und Altruismus geprägten Lebenseinstellung auch immer wie- der seine umfassende Bildung und sprachliche Genauigkeit. Diese findet bei Nestroy mit all seinem hintergründigen Wortwitz eine wunderbare Entsprechung. Die Rolle des Herrn von Lips im „Der Zerissene“, die er neben anderen Rollen beim Theaterspielen verkörperte, ist in lebhafter Erinnerung. Neugierde und Wissensdurst führten Professor Hitzenberger auf zahlreiche Segeltörns und Motorbootfahrten. Der Lesestoff besteht aus Klassikern, aktueller Literatur und nach wie vor medizinischen Artikeln.

Den Zusammenhang herzustellen zwischen jahrzehntelangem Wissen über medizinische Erkenntnisse, Moden, Sackgassen und Erfolgen, viele gefärbt von persönlichen Begegnungen und Erlebnissen und dem aktuellen Stand kultureller, gesellschaftlicher und wissenschaftlicher Bemühungen macht ihm Spaß und ist gleichzeitig ein Quell für uns Jüngere, den er mit Freude und Bereitschaft teilt und mitteilt.

Dem so aktiven und geistig-seelisch so jungen Jubilar wünschen wir Gesundheit und alles Gute für viele Jahre.

Uns Mitarbeitern der $W M W$ und unseren Lesern wünschen wir noch viele medizinische und menschliche Anregungen von ihm.<smiles>CC(C)(C)c1ccccc1</smiles>

Herbert Kurz

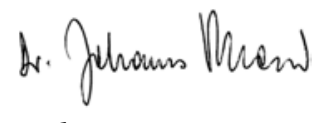

Johannes G. Meran

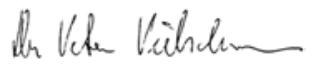

Peter Pietschmann

Redaktion der Wiener Medizinischen Wochenschrift

Das vorliegende Heft der WMW geht in der Konzeption auf die Idee Professor Hitzenbergers zurück und wir freuen uns ihm dieses Heft zum Geburtstag zu widmen.

Der Laudatio sei ein Interview beigefügt, das aus einer freundschaftlichen Unterhaltung beim Heurigen im diesjährigen Oktober unter den Herausgebern der WMW herausdestilliert wurde.

\section{Interview Professor Gerhart Hitzenberger, Oktober 2014}

Herbert Kurz: Lieber Gerhart, was möchtest Du uns darüber erzählen, wie Du in die Medizin gekommen bist?

Gerhart Hitzenberger: Ich war in Wien ins Gymnasium gegangen, wo wir neben anderen zusätzlichen Interessen auch Theater gespielt haben oder Lesungen - von damals verbotenen Autoren - veranstaltet haben. Ich hatte also auch ein großes Interesse an Germanistik und durchaus daran gedacht, Germanistik zu studieren und zusätzlich Schauspieler zu werden. Die andere Option war Medizin. Zur Entscheidungshilfe habe ich mir Doktorarbeiten angesehen. „Die Interpunktion im Werk Wolfram von Eschenbachs“ und Ähnliches war mir dann doch zu langweilig... und die Entscheidung fiel auf die Medizin.
Ich wollte etwas gegen Krebs erfinden - unser Kindermädchen hatte Brustkrebs und das ging mir sehr nah.

Johannes Meran: Deine Eltern waren doch beide Ärzte...

Gerhart Hitzenberger: Ja, mein Vater war sogar ein berühmter Internist, sodass ich ursprünglich dachte, alles nur kein Internist zu werden. Viel später auf Kongressen wurde ich noch gefragt, ob ich nicht verwandt mit dem "Zwerchfell-Hitzenberger" wäre. Mein Vater war ein Pionier in der klinischen Radiologie, er hat den sogenannten „Schnupfversuch“ publiziert, bei dem unter Durchleuchtung die Zwerchfellbewegung beurteilt wird. In Zeiten vieler pulmonaler Prozesse, Narben- und Schwartenbildungen war das klinisch hochrelevant. Mein Vater ist leider zum frühen Opfer der exzessiven Röntgenstrahlung geworden - er starb mit 47 Jahren an einer Leukämie. Meine Mutter war praktische Ärztin.

Peter Pietschmann: Wo hast Du medizinisch angefangen?

Gerhart Hitzenberger: Ich habe als Student, was damals durchaus üblich war, bereits an der 1. Medizinischen Universitätsklinik, die von Prof. Lauda geleitet wurde, unter Oberarzt Keibl, der sich mit Blutkrankheiten beschäftigte, gearbeitet. 
Peter Pietschmann: Was war das Thema Deiner ersten wissenschaftlichen Arbeit?

Gerhart Hitzenberger: Meine erste wissenschaftliche Arbeit hat sich mit der Abstammung der Thrombozyten beschäftigt. Einerseits wurde diskutiert, dass die Thrombozyten von den Megakaryozyten abstammen; andererseits hatte eine deutsche Arbeitsgruppe die Hypothese vertreten, dass es sich bei den Thrombozyten um ausgestoßene Erythrozytenkerne handle. Ich bin im Labor dieser Fragestellung mit speziellen Färbungen (Feulgenfärbung, Hodgkissfärbung) nachgegangen und konnte mikroskopisch eindeutig nachweisen, dass die Thrombozyten von den Megakaryozyten abstammen. Ich hatte diese Arbeiten auf der Station von Oberarzt Keibl durchgeführt. Diesem haben die Ergebnisse sehr gut gefallen und er hatte vorgeschlagen, die Forschungsarbeit bei der Gesellschaft für Innere Medizin vorzustellen. Da ich damals noch Medizinstudent war, wollte Oberarzt Keibl den Vortrag selbst halten; kurz davor hatte ich ihm alle Färbungen noch ganz genau erklären und zeigen müssen.

Herbert Kurz: Wie ging es nach der Promotion weiter?

Gerhart Hitzenberger: Nun, ich bewarb mich an der 1 . Medizinischen Klinik, bekam aber zunächst eine Absage.

Herbert Kurz: Wie muss man sich die früheren leitenden Professoren vorstellen? Das waren doch noch richtige „Götter“ - mich würde interessieren, ob sie wirklich so gut waren, wie ihr Ruhm.

Gerhart Hitzenberger: Natürlich war alles viel autoritärer früher. Aber es gab herausragende Lehrer und Diagnostiker. Ich kann mich an einen Fall erinnern, bei dem Prof. Lauda alleine aufgrund des Tastbefundes der Milz, ihrer Konsistenz und Oberfläche behauptete: „das ist keine Tuberkulose“ und Recht damit hatte. Oder perkutorisch - natürlich hat das alles sehr lange gedauert einen nur $\mathrm{cm}$ großen Lungenherd diagnostizierte.

Und es gab auch immer schon Menschen, die sich trauten, was zu sagen, auch gegenüber dem Vorstand. Dieser Tatsache habe ich ein bisschen meine Anstellung zu verdanken. Bei einer Visite am Samstag-Nachmittag auf C15 hat Prof. Spitzy, er war damals sogenannter „Hilfsarzt“, was heute einem Assistenten entspricht, zu Prof. Lauda gesagt: „Sie sollten den Dr. Hitzenberger anstellen!“ Prof. Lauda, überrascht von der Intervention des Assistenten, fragte: „Und wie soll ich das machen, wenn keine Stelle frei ist?“ Spitzy antwortete keck: „Das müssen Sie wissen, wie das gehen könnte!“ Tatsächlich war es dann so, dass Georg Geyer zwei Jahre nach Amerika ging und ich bekam die Karenzstelle.

Auch sonst wurden die Professoren auch kritisiert. Von Prof. Wenckebach hieß es: „Beim Prof. Wenckebach sind nur die ersten Bänke wach“, weil er so leise sprach in der Vorlesung.
Johannes Meran: Was kannst Du über die Veränderung der Strukturen über die Jahrzehnte hinweg sagen?

Gerhart Hitzenberger: Alles hat viel länger gedauert. Wir haben lange Visiten gehabt, wir haben selbst die Laborwerte bestimmt, Harn, Zucker und so weiter, wir haben selbstverständlich selbst die Präparate gefärbt, mikroskopiert. Die Patienten lagen auch meist lange stationär. Auch Spezialisierungen haben sich langsam entwickelt. Ich ging ja zur Ausbildung in Klinischer Pharmakologie in die USA, nach Ann Arbor, Michigan. Dort lernte ich auch Stevo Julius (er schrieb später „Adventures in Hypertension") kennen und begann mich mit Hypertonie zu beschäftigen. In diesem Krankenhaus hatten wir einmal einen Fall eines Patienten mit einer schweren Endokarditis, die nicht in den Griff zu bekommen war. Der zuständige Prof. Carr fragte mich „Was würden Sie machen?“. Ich habe die „Spitzy-Therapie“ empfohlen, die aus 3 mal 20 Mio. Einheiten Penizillin G bestand. Sie hat geholfen.

Herbert Kurz: Du überblickst einen langen Zeitraum Medizin - sind die Fortschritte wirklich so groß und spürbar für die Patienten?

Gerhart Hitzenberger: Ich glaube schon. Heute kann man zum Beispiel etwas gegen Rheuma tun. Auch die Fortschritte in der Chirurgie und bei den Implantaten, Gelenksersatz usw. sind ungeheuer und bringen spürbare Verbesserungen für die Menschen.

Johannes Meran: Und die jüngsten Durchbrüche in der Behandlung der CML... und der Morbus Hodgkin ist heute heilbar!

Gerhart Hitzenberger: Ja, wir haben während meiner Studentenzeit den Patienten mit Blutkrebs frisches Rinderknochenmark zu essen gegeben... Sonst gab es kaum etwas.

Peter Mikosch: Was halten Sie selbst für die wichtigen Beiträge, die Sie für den Fortschritt der Medizin in Wien beigetragen haben?

Gerhart Hitzenberger: Ich habe an der 1. Med. erstmals Studien mit Statistik veröffentlicht. Und ich habe zusammen mit Prof. Spitzy die erste Ethikkommission noch unter Prof. Deutsch gegründet.

Johannes Meran: Heute kann man sich gar nicht mehr vorstellen ohne Statistik und ohne Ethikvotum eine Studie zu beginnen. Du hast ja dann auch viele Jahre in der Ethikkommission mitgewirkt und letztlich die heutigen Standards miterarbeitet.

Peter Pietschmann: Was würdest $\mathrm{Du}$ einem jungen Mediziner für seine Ausbildung raten? 
Gerhart Hitzenberger: Ich würde ihm raten, sich sehr um die anvertrauten Patienten zu kümmern. Sehr wichtig ist es auch Mentoren zu haben. Meine Mentoren waren vor allem Prof. Spitzy und Prof. Geyer. Ich erinnere mich noch, wie mir Prof. Geyer viel über die Perkussion und Auskultation gezeigt hat.

Peter Pietschmann: Damals wie heute wird den Ärzten der „Triple Track“, das heißt gleichzeitige Tätigkeiten in
Patientenversorgung, Lehre und Forschung abverlangt. Ist das Deiner Erfahrung nach überhaupt möglich?

Gerhart Hitzenberger: Ich glaube, es geht nicht.

Herbert Kurz: Als was siehst Du Dich am ehesten, als Forscher, Lehrer oder Arzt?

Gerhart Hitzenberger: Als Arzt. 